\title{
Radiation-tolerant silicon detectors for the LHC Phase-Il upgrade and beyond: An overview of RD50 activities
}

\author{
Jennifer Ott ${ }^{* \dagger}$ \\ Helsinki Institute of Physics, Gustaf Hällströmin katu 2, FI-00014 University of Helsinki, \\ Finland \\ Aalto University School of Electrical Engineering, Tietotie 3, FI-02150 Espoo, Finland \\ E-mail: jennifer.ott@helsinki.fi
}

At the LHC Phase-II Upgrade foreseen for 2027, the particle densities and radiation levels will increase by roughly an order of magnitude compared to the present LHC conditions, and the siliconbased inner tracking systems have to be able to withstand fluences of up to $2 \times 10^{16} \mathrm{n}_{e q} / \mathrm{cm}^{2}$. To mitigate the higher pileup associated with the significant increase in instantaneous luminosity at the HL-LHC, dedicated timing detectors are employed.

Within the CERN RD50 Collaboration, a large R\&D program has been underway for more than a decade across experimental boundaries to develop silicon sensors with sufficient radiation tolerance for HL-LHC tracking and timing detectors. This challenge is approached simultaneously from different angles: Collaboration activities range from defect characterization and modeling to sensor development and the integration of sensors into full detector systems.

One of the main objectives of the RD50 Collaboration is to improve understanding of the connection between the macroscopic sensor properties, such as radiation-induced increase of leakage current and trapping, and the microscopic properties at the defect level. An example of this is the acceptor removal phenomenon in p-type silicon, which is affecting especially the low-gain avalanche detectors developed for fast timing applications. With increasing fluences, radiationinduced phenomena on a sensor level become increasingly complex, and call for advanced techniques and strategies to identify the mechanisms behind the observed changes in material properties. Furthermore, at very high radiation levels the differences in radiation damage caused by different types of radiation are highlighted, which complicates the definition and scaling of radiation damage.

In this paper, we summarize the silicon pixel and timing detector upgrades of the LHC experiments and report on recent developments in novel silicon detector technologies, most importantly 3D detectors and low-gain avalanche detectors. Scaling of radiation damage based on leakage current and $1 \mathrm{MeV}$ neutron equivalents is challenged. We also comment on considerations for silicon detectors in future collider experiments, where tracker detectors may be exposed to fluences of up to $7 \times 10^{17} \mathrm{n}_{e q} / \mathrm{cm}^{2}$.

The 28th International Workshop on Vertex Detectors - Vertex2019

13-18 October, 2019

Lopud, Croatia

\footnotetext{
* Speaker.

${ }^{\dagger}$ On behalf of the RD50 Collaboration
} 


\section{Introduction}

Silicon tracking detectors form the core of all large collider physics experiments. The RD50 Collaboration at CERN [1] has been studying silicon detectors since the early 2000s, with the mandate of developing radiation-hard semiconductor devices for the upgrades of the LHC experiments towards the High-Luminosity LHC, and for future high-luminosity colliders. The RD50 Collaboration unites around 360 experts from 60 institutes in 22 countries, including scientists from all large LHC experiments, as well as the Belle-II experiment at the SuperKEKB accelerator in Japan. Research topics extend from Defect and Materials Characterization over Detector Characterization to the development of New Structures and study of Full Detector Systems, while aiming to combine experimental data with Simulation.

This article gives an overview of material aspects and new technologies of silicon detectors for the HL-LHC era that are developed and studied within the RD50 Collaboration. Advances and open questions in device and material characterization, and challenges in the evaluation and scaling of radiation damage at very high fluences are discussed. Finally, challenges and requirements for silicon detectors in different future collider proposals beyond the HL-LHC are addressed.

\section{Silicon detectors for the High-Luminosity LHC}

The upgrade of the LHC to the HL-LHC, planned to commence operation in 2027, brings an increase of instantaneous luminosity by a factor of 5 compared to the LHC, to at least $5 \times 10^{34}$ $\mathrm{cm}^{-2} \mathrm{~s}^{-1}$, and an increase of integrated luminosity from 400 to $3000-4000 \mathrm{fb}^{-1}$. This concurs with the increase of radiation levels to total fluences of $>2 \times 10^{16} \mathrm{n}_{e q} / \mathrm{cm}^{2}$ at the innermost silicon detector layers. In addition, an increase in the number of primary vertices per bunch crossing (pileup) from 50 to about 200 is predicted. The detectors of the large general-purpose experiments at the LHC, ATLAS and CMS, will be upgraded for the HL-LHC period, including a complete renewal of the silicon inner trackers [2, 3]. These so-called Phase-II upgrades also include the installation of dedicated timing detectors in order to reduce pileup and increase precision in particle identification $[4,5,6]$. To provide detectors able to cope with the harsh radiation environments and simultaneously provide an improved performance, e.g. in terms of spatial and timing resolution, new approaches in material and device engineering are taken, and new detector technologies have been developed.

\subsection{Establishing p-type silicon}

Traditionally, silicon detectors have been fabricated on n-type $\mathrm{Si}$, due to its availability in sufficient quality and high resistivity, which provides low leakage currents and power consumption and enables the operation of the detector in full depletion at lower voltages. Furthermore, the majority carriers in n-type $\mathrm{Si}$ are electrons, which possess a higher mobility than holes, and for example iron contaminants are less recombination-active in n-type material than in boron-doped p-type silicon [7].

The effects of various types of radiation on n-type Si have been studied thoroughly over many years. Most prominently, n-type silicon sensors are observed to undergo compensation and further inversion of the space charge polarity, a phenomenon often referred to as "type inversion": the full 
depletion voltage passes through a minimum after a certain fluence, but increases again linearly afterwards, while the effective space charge of the bulk becomes negative, corresponding to p-type silicon.

The silicon detectors currently installed in the large LHC experiments are based on n-type substrates. However, the CMS and ATLAS experiments already transitioned to read-out from the $\mathrm{n}^{+}$electrode, collecting signals of negative polarity, in the Phase-I upgrade of their inner tracker pixel detectors. For the future - upon completion of the phase-II upgrades of ATLAS and CMS to the HL-LHC in 2024-2026, as well as the LHCb and ALICE upgrades to be completed by 2020 all large LHC experiments have named p-type silicon sensors as baseline for their pixel and timing detectors $[2,3,8,9]$. While this is predicted to improve radiation hardness of the detectors and to avoid the effective bulk type inversion observed for n-type silicon, some radiation effects and their mitigation in p-type silicon are not fully understood, as discussed in the following sections.

\subsection{Planar pixel sensors}

The principle of planar pixel technology has remained rather similar over the years. The emphasis of research and development has been on the decrease of pixel pitch to increase granularity and improve spatial resolution, and improve coverage and hit efficiency by reduction of dead area at the detector edges. For the HL-LHC, the pixel pitch in ATLAS and CMS is reduced from the $100 \times 150 \mu \mathrm{m}^{2}$ or $50 \times 450 \mu \mathrm{m}^{2}$ in the current detectors, respectively, to $50 \times 50 \mu \mathrm{m}^{2}$ or $25 \times$ $100 \mu \mathrm{m}^{2}$ [2,3], matching the geometry of the new RD53 readout chip developed jointly by the experiments [10].

An additional challenge lies in the transition from n- to p-type silicon with negative-polarity readout from the segmented $\mathrm{n}^{+}$implant, where the signal is now read out at the $\mathrm{p}-\mathrm{n}$ junction and not the ohmic contact, as would be the case in $\mathrm{n}^{+}$-in- $\mathrm{n}$ sensors. This is motivated by the advantage of positioning the onset of the depletion region (in case the sensor would be operated in underdepletion at any stage), as well as the maximum of the electric field, at the segmented readout electrode.

In this configuration, the positive oxide charge of the traditional $\mathrm{SiO}_{2}$ dielectric is detrimental, and without corrective action it would lead to the effective connection of all segments to each other, causing loss of spatial resolution. To ensure electrical insulation, the addition of an implant between the pixels (known as p-spray or p-stop) compensating for the positive oxide charge has been established [11, 12]. As an alternative to these insulating implants, the use of negative-charge dielectrics, such as aluminium oxide, has been proposed [13, 14].

\subsection{D sensors}

3D sensors [15] have been discussed both for tracking detectors as an alternative to planar pixel sensors, and for timing applications. As opposed to the $\mathrm{p}^{+}$and $\mathrm{n}^{+}$implants being placed in (segmented) sheets on opposite sides of the silicon wafer as in the case of planar strip and pixel sensors, in 3D sensors both types of electrodes are formed by alternating groups of vertical columns (Fig.1a). This connects the required drift distance for charge carriers to the pitch of the columns, instead of the bulk thickness in a planar device. Therefore the collection of a sufficiently large signal is less affected by trapping and recombination centers formed during irradiation of 
the device, ensuring very high efficiencies and resolution even at HL-LHC fluences (Fig.1b) [16, 17]. In addition, shorter drift distances, in combination with a high electric field between the electrodes, also lead to faster drift times, which enables timing resolution down to around $75 \mathrm{ps}$ with unirradiated 3D sensors [18].
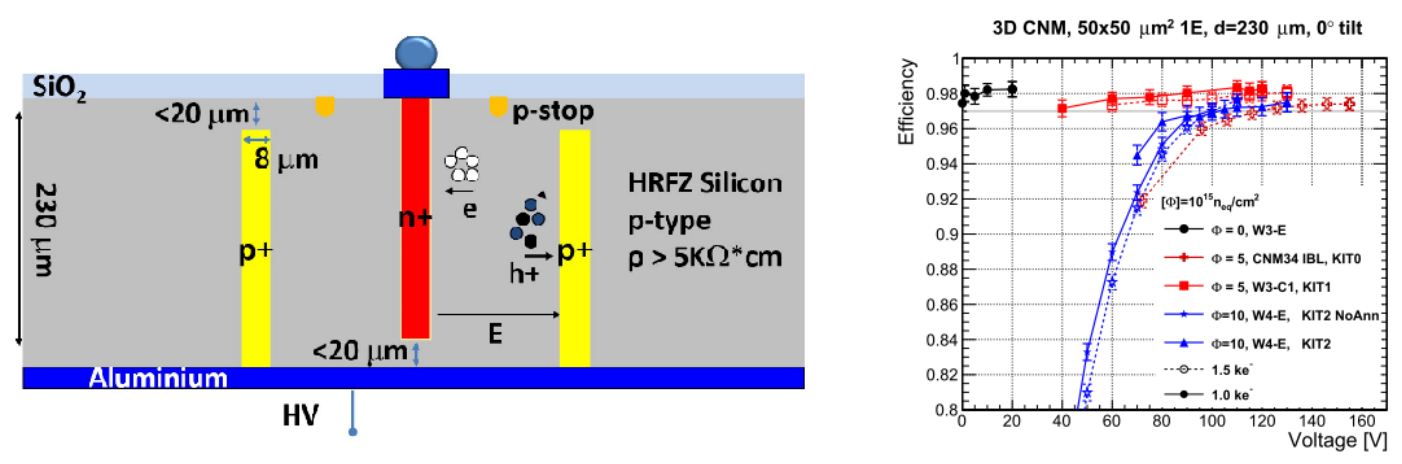

Figure 1: a) Left: Schematic cross-section of a small-cell 3D sensor [19], b) right: Hit efficiency as function of bias voltage for an irradiated 3D sensor [17].

3D sensors have already seen use in the insertable barrel pixel layer in the ATLAS inner tracker, and are included in the inner layers of both CMS and ATLAS Phase-II upgrade designs $[2,3]$. Current research in 3D sensors is focusing on small-cell sensors to meet future requirements for finer granularity in vertexing detectors, and to improve the understanding signal formation and properties for different particle track angles especially in highly-irradiated sensors [17, 18, 19].

\subsection{LGAD sensors}

The current concepts for the endcap timing layers in both experiments (the CMS timing detector also includes a scintillator+SiPM -based barrel region) feature silicon low-gain avalanche diodes (LGADs) [4, 5]. As its name indicates, the LGAD is a silicon sensor with low to moderate gain on the order of 5-100, compared to other multiplication based silicon devices such as silicon photomultipliers or avalanche photodiodes. State-of-the art LGADs are implemented on p-type substrates with an $\mathrm{n}^{+}$implant on the readout side, with the gain provided by an additional borondoped multiplication layer below the top electrode, as shown in Fig.2. Doping oncentration, depth, width (i.e. extension into the bulk) and side termination of the gain layer directly influence not only the gain of the sensor, but also other properties such as signal rise time, leakage current, and breakdown behavior. The fast and high signal formed by the local secondary ionization avalanche at the gain layer is further supported by considerably low active thicknesses of $35-50 \mu \mathrm{m}$ in most recent productions, which provide a higher electric field than in thicker devices at the same bias voltage. To date, timing resolution of 20-30 ps can be reached with LGAD sensors.

The main challenges in LGAD sensors are their radiation hardness in terms of gain, and the fill factor of segmented LGADs.

The fill factor, i.e. the ratio of active area over the total geometrical area of the sensor, is largely determined by the design of the inter-pad regions. In the standard LGAD design, the device consists of segments separated from each other by a non-gain region including the junction termination 
extension and p-stop insulation, thus the gain layer is segmented. For particle hits incident in the interpad region, efficiency and timing resolution are inferior. To improve the fill-factor, in planar traditional LGADs, the width of the non-gain interpad regions is aimed to be minimized through adjustment of the JTE and p-stop implants, while avoiding an early breakdown of the sensor between pads. Presently, the lowest interpad distances for stable sensor operation range from 30 to $70 \mu \mathrm{m}$. This distance can potentially be reduced by insulating the segments with trenches etched into the silicon, as presented in Fig.3 [20, 21].

In the so-called inverse LGAD (iLGAD) concept, on the other hand, the n+ electrode and the underlying gain layer are kept uniform entirely, and the segmentation is instead implemented at the $\mathrm{p}^{+}$ohmic contact electrode [22, 23]. Another related line of research are capacitively (AC) coupled LGADs with a resistive sheet under the electrodes, also allowing for a uniform gain layer [24].
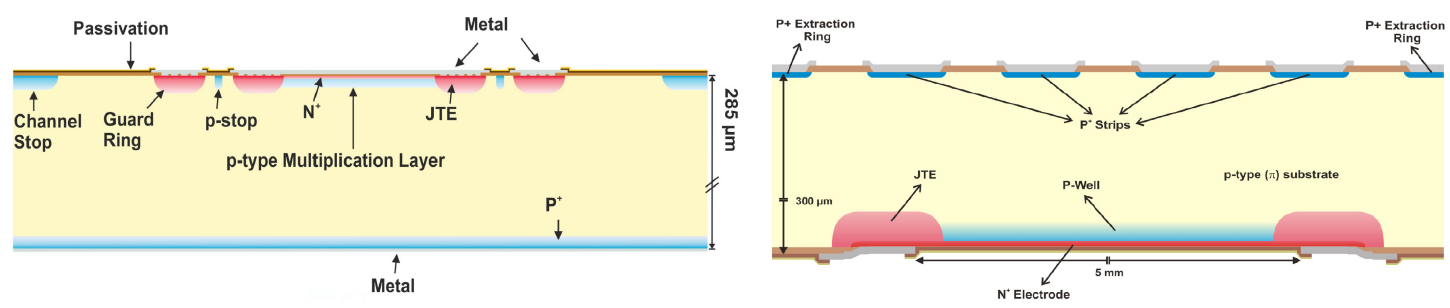

Figure 2: Left: Schematic cross-section of a standard LGAD [23], right: Schematic of a p-in-p iLGAD [22].
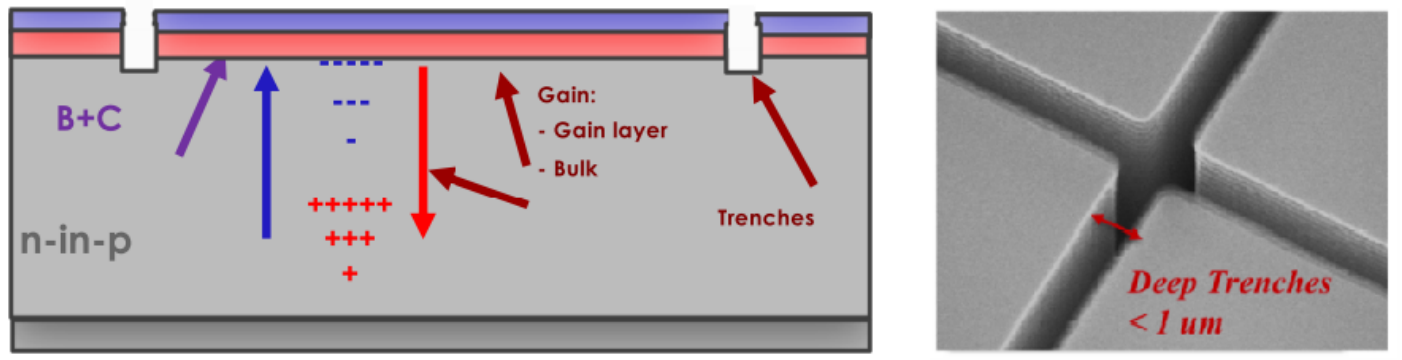

Figure 3: Left: schematic of a trench-isolated LGAD [20], right: image of a trench-isolated LGAD [21].

The most prominent radiation effect in LGAD sensors is the drastic reduction of gain achievable in the device, and shift of the same gain to higher bias voltages with irradiation (Fig.4a). This is attributed to a decrease in the concentration of active boron dopants in the gain layer (Fig.4b)[25, 26, 27], a manifestation of the so-called acceptor removal effect, to be discussed further in Section 3.2. In addition to adjusting other properties of the gain layer as mentioned above, co-doping of the gain layer with carbon has been demonstrated to enhance the retention of the gain layer in irradiation: when lattice defects are created by incident radiation, some of the interstitial sites are filled by carbon atoms instead, keeping a larger fraction of boron dopants electrically active [27]. A slightly different approach, where the gain layer is fabricated using gallium as dopant instead of boron, sometimes also in combination with carbon, requires further studies [27, 28]. 

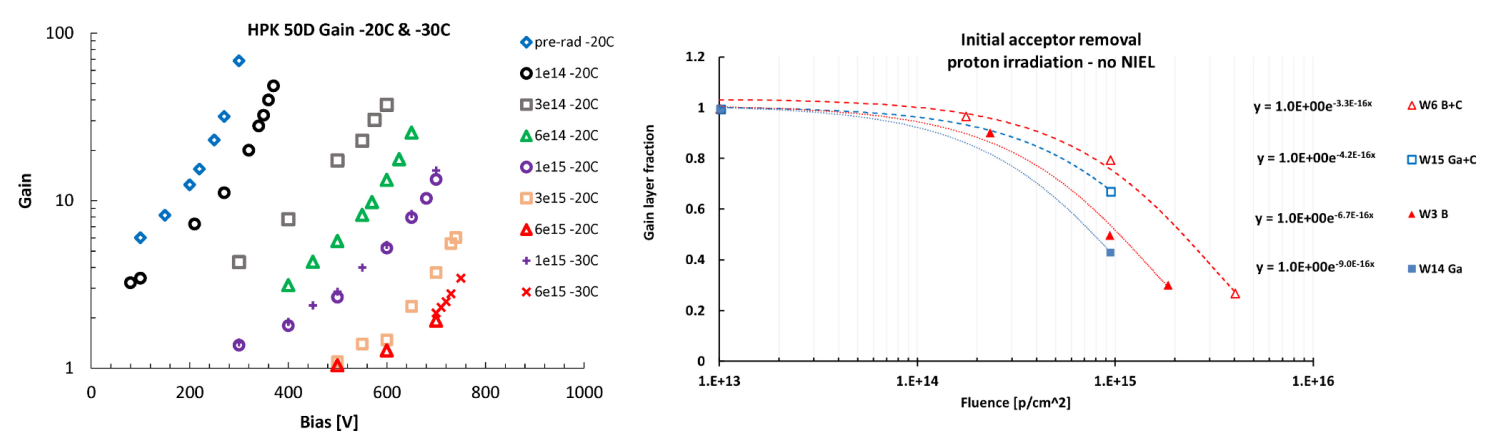

Figure 4: a)(left) Gain loss in an HPK LGAD irradiated with neutrons [26]. b) (right) Deactivation of the gain layer doping in FBK LGADs [27].

\subsection{Up the sleeve: CMOS sensors}

Depleted and high-voltage CMOS detectors[29], also referred to as monolithic active pixel sensors (MAPS) are an attractive technology for large-scale silicon particle detectors: the frontend ASIC can be integrated on the sensor chip, therefore removing the need for expensive flip-chip bonding of separate sensor and ASIC chips as would be done in a hybrid detector. The integration of active sensor volume and front-end onto the same silicon chip also reduces the total material budget of the detector. Furthermore, CMOS is an established industrial technology and allows for the use of large foundries and large wafers in detector fabrication, which reduces the cost of production especially for higher production volumes.

The drawback of CMOS sensors is their lower radiation tolerance compared to other sensor technologies; signal collection and efficiencies are significantly degraded at fluences on the order of $1 \times 10^{15} \mathrm{n}_{e q} / \mathrm{cm}^{2}$ [30]. Due to this, CMOS sensors are at the moment not considered for the most challenging radiation environments, i.e. they are not included in the designs of the initial ATLAS and CMS Phase-II upgrades. However, they are kept as an option for a replacement of the outermost pixel layer in the ATLAS inner tracker [31]. As the first large collider experiment, the ALICE experiment will feature 180nm CMOS technology on p-type Si in the inner tracking system after its upgrade for the LHC Run3 [8]. For a further upgrade of the detector to continue operation during the HL-LHC, the use of very thin 65nm CMOS silicon sensors on large wafers $(300 \mathrm{~mm})$ is being investigated.

It is expected that CMOS sensor technology will play a strong role in experiments at future colliders, where even larger areas need to be covered with silicon detectors and excellent spatial resolution at comparatively low cost is required.

\section{Device, material and defect characterization}

Identifying and understanding the microscopic effects responsible for properties observed on the sensor level is crucial for the successful operation, optimization and further development of silicon detectors. A wide range of characterization methods from the fields of electronics, micro- and nanofabrication, and spectroscopy is available. At all stages of characterization, simulations are a valuable tool for the linking of experimental results and theoretical predictions, and to provide estimations or predictions on material and device properties[32]. Even detector design and processing 
can first be evaluated by simulation, to increase the probabilities for costly detector fabrication to deliver the desired properties. Both commercial (e.g. Synpsys Sentaurus TCAD, Silvaco ATLAS) and open-source software packages (e.g. Weightfield2, KDetSim) are available.

While e.g. standard CV/IV measurements have changed very little over the years, the Transient Current Technique (TCT), where charge injection is achieved by red or IR laser (commonly wavelengths of 660 and $1064 \mathrm{~nm}$, respectively) to study the shape of the electric field and the collection of charges [33], has seen a series of developments. In addition to illuminating the front and back surfaces of the sensor, illumination from the sensor edge (edge-TCT, e-TCT) allows direct probing of charge collection and the electric field in the device as a function of depth [34]. More recently, TCT equipment based on nonlinear two-photon absorption (TPA) has been established a signal is induced only in a small region of 1-10 um around the laser focal point, where the intensity is high enough for absorption of two photons and generation of electron-hole pairs [35, 36]. Electrical characterization with good spatial resolution as provided by TPA-TCT is invaluable for the study of the thinner and more finely segmented silicon devices required in future detectors.

On the microscopic scale, the goal in characterization lies on the correlation of electrical properties with material structure, impurity concentrations and defects. Scanning Electron and transmission Electron Microscopy (SEM and TEM, respectively) provide information about material and device topography, to some extent also on elemental composition and stoichiometry. Charge carrier recombination lifetimes can be studied by Microwave-probed photoconductance (decay) (PC / uPCD). The chemical composition of samples can be analysed e.g. by Secondary Ion Mass Spectrometry (SIMS), while specific electrically active defect levels can be identified and quantified by Deep Level Transient Spectroscopy (DLTS), Thermally Stimulated Currents (TSC) and Fourier Transform Infrared Spectroscopy (FTIR). An overview of the most significant electrically active defects in silicon is shown in Fig.5, along with a schematic representation of the commonly observed effects of radiation on the sensor level.
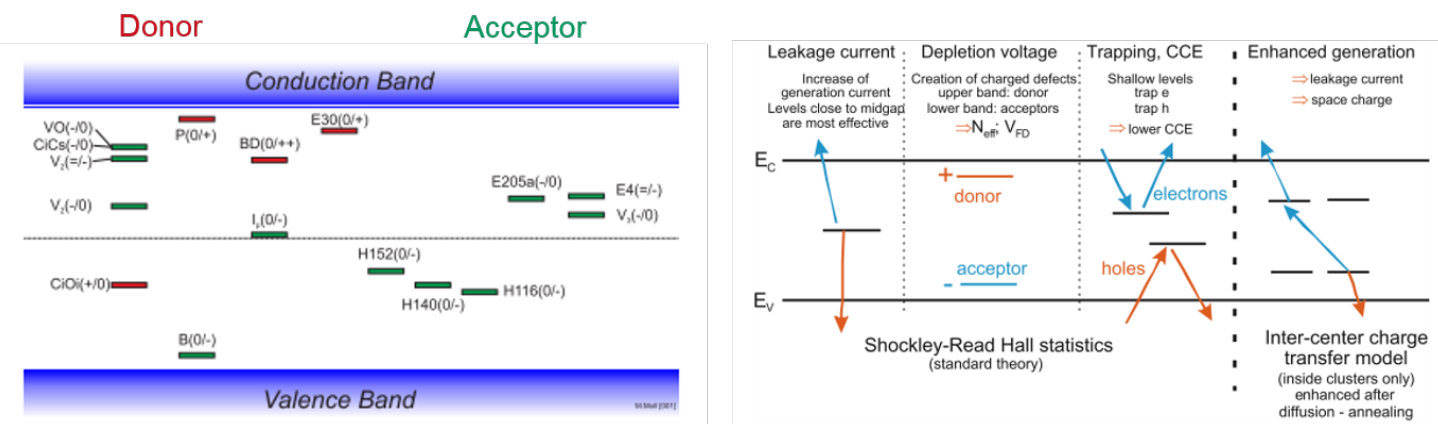

Figure 5: a) (left) Overview of defect levels [37]. b) (right) Macroscopic effects of radiationinduced defects [38].

\subsection{The Hamburg model, NIEL scaling and acceptor removal - the challenge of quantification and scaling of radiation damage}

In recent years, RD50 has been closely involved with the organization of radiation effects workshops, where radiation damage and its impact on the operating silicon detector systems of the large LHC experiments has been discussed [39, 40]. These workshops and the results presented 
therein have provided a large amount of data for the validation and improvement of radiation damage models developed largely within the RD50 Collaboration.

In the present conditions at the LHC detector systems - fluence range between $1 \times 10^{14} \mathrm{n}_{e q} / \mathrm{cm}^{2}$ and $1 \times 10^{15} \mathrm{n}_{e q} / \mathrm{cm}^{2}$ and planar n-type sensors - the experimental data is in good agreement with the Hamburg model, which describes the evolution of leakage current, depletion voltage and other properties as function of fluence and annealing.

At extreme fluences of $>1 \times 10^{16} \mathrm{n}_{e q} / \mathrm{cm}^{2}$, however, the validity of present models is not fully verified, and a saturation of radiation effects at very high fluences has been observed (examples in Fig.6[41, 42]. To elucidate the mechanism behind this and its consequences for detector fabrication and operation, more extensive studies, both experimental and in simulation, are required.
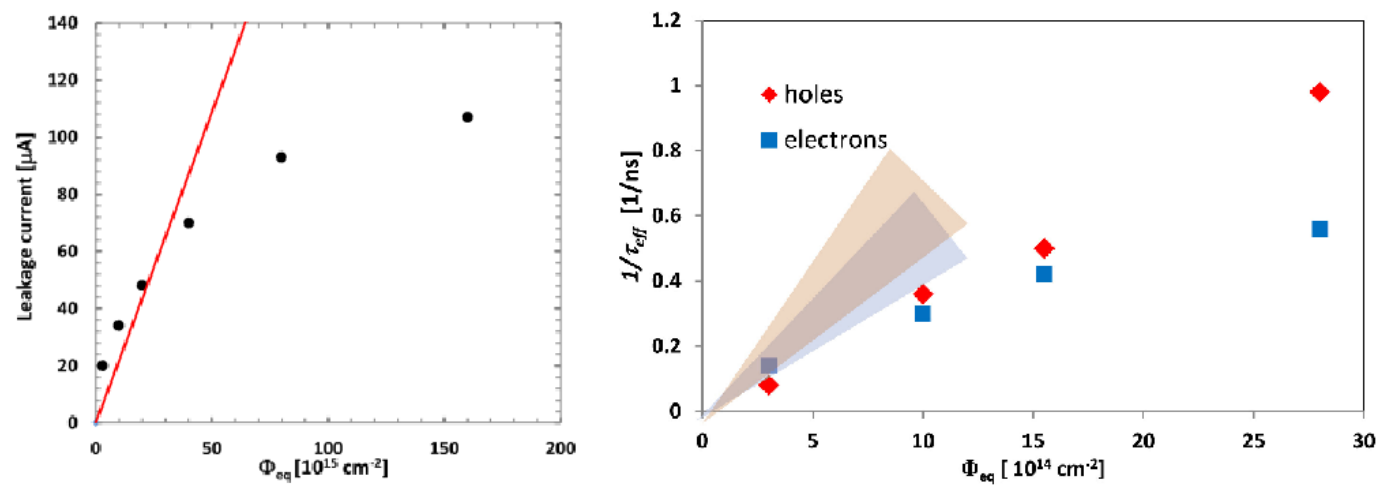

Figure 6: a) (left) Saturation of leakage current at very high fluence [41]. b) (right) Saturation of trapping times for electrons and holes [42].

In order to compare the effects of different types of radiation, the concept of scaling different radiation types and energies to non-ionizing energy loss (NIEL) as $1 \mathrm{MeV}$ neutron equivalents [43] has become established. Recently, it was reminded that the use of this unit in itself introduces considerable uncertainties through the determination of cross-sections and displacement energies [44]. Furthermore, the scaling factors used for this purpose, also known as hardness factors, are based on leakage current densities, and as discussed in the following, this may be not the most suitable measure of radiation damage, depending on the conditions and sensor type.

Future detectors, relying on different technologies and materials as introduced in the previous sections, call for or additional parameters, or slightly different angles of view, to quantify radiation damage:

Lower leakage currents reduce power consumption and limit the risks of breakdown of the device, as well as thermal runaway in irradiated detectors. However, as silicon detector technologies advance and device engineering is improved, leakage current is not anymore the main (or the only) limiting factor.

In addition to the full depletion voltage of the detector, different aspects of charge collection with bias voltage become more relevant to monitor: the operation of the detector in an underdepleted state, and degradation of the total collected charge with irradiation may be deemed tolerable, as long as the sensor still provides a sufficient signal (with a satisfactory signal-to-noise ratio) to the corresponding front-end and readout electronics. The trend to smaller pitches in segmented 
detectors is not only motivated by an improvement in spatial resolution as such, but also to keep the pitch below (or close to) the charge collection length, which is decreased by radiation-induced charge carrier trapping.

Finally, the phenomenon of acceptor removal, i.e. the dislocation and consequent deactivation of boron dopant atoms in p-type silicon, has been observed most prominently in LGADs, which rely on a p-doped layer to provide gain. In this case, deactivation of boron dopants manifests itself as a significant reduction in gain with irradiation (Fig.4a). Detectors relying on charge multiplication suffer from a decrease in gain with irradiation, which impacts their operation far more than a linear and better understood - increase in leakage current. Consequently, the decrease in gain, or rate of the underlying acceptor removal, are more relevant meters for the quantification of radiation damage in such devices. Figure $4 \mathrm{~b}$ indicates further that the deactivation of the gain layer in proton irradiation is described best without the use of NIEL scaling hardness factors.

Acceptor removal is not restricted to LGADs; it is seen to various extents in most types of ptype silicon, including epitaxial Si diodes as shown in Fig.7. TSC measurements indicate the theory that the formation of $\mathrm{B}_{i} \mathrm{O}_{i}$ defects (a complex of interstitial boron and interstitial oxygen) correlates with boron acceptor removal, as the defect prevents the return of the B atom into its electrically active, substitutional state $\left(\mathrm{B}_{s}\right)$ as shallow acceptor [45]. However, further work is required to form a full understanding of the microscopic defect formation kinetics causing acceptor removal[46].

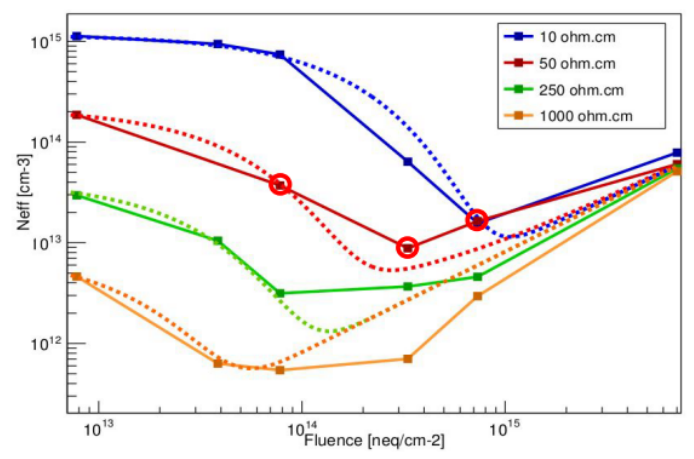

(a)

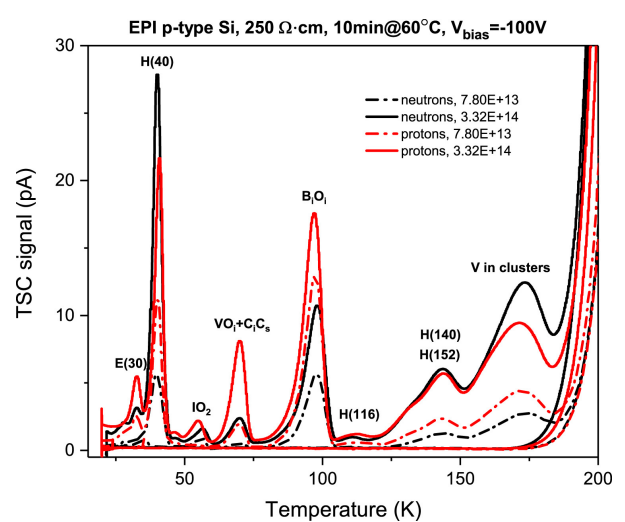

(b)

Figure 7: a) Left: $\mathrm{N}_{\text {eff }}$ as function of proton irradiation fluence for epitaxial silicon diodes [47], b) right: TSC spectra for epitaxial pad diodes after proton (red) and neutron (black) irradiation [45].

\section{Looking towards future colliders}

The coming years are expected to bring decisions for the initiation of major particle collider projects beyond the LHC/HL-LHC. Several candidates for future colliders have been proposed, among them scenarios of both circular and linear accelerators, hadron as well as lepton collisions. Initial proposals for all future collider experiments continue to rely on silicon detectors for vertexing and tracking systems; however, the limitations on silicon detector technologies may differ significantly e.g. in hadron and lepton colliders. Therefore, future research on silicon detectors will be directed to a large extent by the final choice(s) of collider environment and physics cases. 


\subsection{Hadron colliders}

In future hadron colliders, new physics discoveries are sought by an even further increase of center-of-mass energies and luminosities compared to the HL-LHC. For the hadron collision stage of the Future Circular Collider (FCC-hh), with a circumference of up to $100 \mathrm{~km}$ and collision energies of $100 \mathrm{TeV}$ in proton-proton collision, fluences of up to $7 \times 10^{17} \mathrm{n}_{e q} / \mathrm{cm}^{2}$ on inner and/or forward silicon detector layers have been estimated [48]. These immense fluences, along with high occupancy and pileup, would set a very high threshold for silicon sensor and readout technologies, design, and material choices. Although silicon sensors have been shown to survive fluences of up to $3 \times 10^{17} \mathrm{n}_{e q} / \mathrm{cm}^{2}$ [41, 49], ensuring the retention of a sufficient signal and/or timing capabilities below 20 ps poses an extreme challenge on silicon detector technologies.

\subsection{Lepton colliders}

Several proposals for lepton colliders have been presented: the Compact Linear Collider (CLIC) and the International Linear Collider (ILC) featuring linear acceleration of leptons, and on the other hand the Circular Electron-Positron Collider (CEPC) and a lepton stage of the FCC (FCC-ee) as circular accelerators.

In lepton colliders with their absence of significant pileup as well as QCD background found in hadron colliders, the reduction of multiple scattering by very low material budget in combination with very high detector granularity is the key requirement for the inner vertex detectors layers. For the vertex detectors in the CLIC [50] and ILC, for example, spatial resolutions of $3 \mu \mathrm{m}$ and mass thicknesses of $0.15-0.3 \% \mathrm{X}_{0}$ are pursued. On the other hand, fluences delivered by charged particles on the silicon detectors are expected to be lower than in the first runs of the LHC, with initial estimations of only around $1 \times 10^{14} \mathrm{n}_{e q} / \mathrm{cm}^{2}$ consisting mainly of light charged particles and bremsstrahlung radiation. For such a detector, thin depleted CMOS sensors or hybrid pixels are expectedly the dominant sensor technologies.

\section{Summary}

Research and development of silicon detectors are well underway towards the HL-LHC. In order to deliver excellent detector performance despite the harsh radiation environments and pileup, the experiments will feature new detector technologies, such as LGAD and 3D sensors, alongside improved planar devices. As fluences increase and detector technology are more specialized, also the quantification and scaling of radiation effects in these sensors becomes more complex. In the p-type substrates baselined for the HL-LHC silicon detectors, the acceptor removal phenomenon is yet to be fully understood. To a growing extent, the development of silicon detector technologies for use in future collider experiments also calls for extensive RD efforts. The RD50 Collaboration remains committed to the study of radiation-tolerant silicon detectors through different approaches, and continues to provide a forum for the presentation of new results and the development of new ideas. 


\section{Acknowledgments}

J. Ott would like to thank the members of the RD50 Collaboration for many fruitful discussions during workshops, and acknowledges the Vilho, Yrjö and Kalle Väisälä Foundation of the Finnish Academy of Science and Letters for financial support.

Most irradiation campaigns in RD50 have been performed under the transnational access agreement funded by the European Union's Horizon 2020 Research and Innovation program under Grant Agreement no. 654168 (AIDA-2020).

\section{References}

[1] RD50Collaboration, "RD50 Homepage." http://rd50.web.cern.ch/rd50/.

[2] ATLASCollaboration, "Technical Design Report for the ATLAS Inner Tracker Pixel Detector." CERN-LHCC-2017-021, ATLAS-TDR-030, 2017.

[3] CMSCollaboration, "The Phase-2 Upgrade of the CMS Tracker, Technical Design Report." CERN-LHCC-2017-009,CMS-TDR-0141, 2017.

[4] CMSCollaboration, "A MIP Timing Detector for the CMS Phase-2 Upgrade." CERN-LHCC-2019-003, CMS-TDR-020, 2019.

[5] ATLASCollaboration, "Technical Proposal: A High-Granularity Timing Detector for the ATLAS Phase-II Upgrade.” CERN-LHCC-2018-023, LHCC-P-012, 2018.

[6] S. Otero, LGAD and 3D as Timing Detectors, Proceedings of Science (Vertex2019) (2020) .

[7] L. Geerligs and D. Macdonald, Base Doping and Recombination Activity of Impurities in Crystalline Silicon Solar Cells, Progress in Photovoltaics: Research and Applications 12 (2004) 309.

[8] ALICECollaboration, "Technical Design Report for the Upgrade of the ALICE Inner Tracking System.” CERN-LHCC-2013-024, ALICE-TDR-017, 2013.

[9] LHCbCollaboration, "LHCb Tracker Upgrade Technical Design Report." CERN-LHCC-2014-001, LHCB-TDR-015, 2014.

[10] RD53Collaboration, “The RD53A Integrated Circuit, Version 3.42.” CERN-RD53-PUB-17-001, 2018.

[11] J. Schwandt, CMS Pixel detector development for the HL-LHC, Nuclear Instruments and Methods in Physics Research A 924 (2019) 59.

[12] A. Ducourthial, M. Bomben, G. Calderini, R. Camacho, L. D'Eramo, I. Luise et al., Performance of thin planar n-on-p silicon pixels after HL-LHC radiation fluences, Nuclear Instruments and Methods in Physics Research A 927 (2019) 219.

[13] J. Härkönen, J. Ott, M. Mäkelä, T. Arsenovich, A. Gädda, T. Peltola et al., Atomic Layer Deposition (ALD) grown thin films for ultra-fine pitch pixel detectors, Nuclear Instruments and Methods in Physics Research A 831 (2016) 2.

[14] J. Ott, A. Gädda, S. Bharthuar, T. Naaranoja, L. Martikainen, S. Kirschenmann et al., Processing of $A C$-coupled $n$-in-p pixel detectors on $M C z$ silicon using atomic layer deposited aluminium oxide, Nuclear Instruments and Methods in Physics Research A,https://doi.org/10.1016/j.nima.2019.162547 
[15] S. Parker, C. Kenney and J. Segal, 3D - A proposed new architecture for solid-state radiation detectors, Nuclear Instruments and Methods in Physics Research A 395 (1997) 328.

[16] D. V. Furelos, M. Carulla, E. Cavallaro, F. Förster, S. Grinstein, J. Lange et al., 3D sensors for the HL-LHC, Journal of Instrumentation 12 (2017) C01026.

[17] J. Lange, G. Giannini, S. Grinstein, M. Manna, G. Pellegrini, D. Quirion et al., Radiation hardness of small-pitch 3D pixel sensors up to a fluence of 3 e16 neq/cm2, Journal of Instrumentation 13 (2018) P09009.

[18] G. Kramberger, V. Cindro, D. Flores, S. Hidalgo, B. Hiti, M. Manna et al., Timing performance of small cell 3D silicon detectors, Nuclear Instruments and Methods in Physics Research A 934 (2019) 26.

[19] E. Currás, J. Duarte-Campderrós, M. Fernández, A. García, G. Gómez, J. González et al., Study of small-cell 3D silicon pixel detectors for the high luminosity LHC, Nuclear Instruments and Methods in Physics Research A 931 (2019) 127.

[20] N. Cartiglia, "Tracking particles at fluences 1E16-1E17 n/cm2.” 14th Trento Workshop on Advanced Silicon Radiation Detectors, 2019.

[21] G. Borghi, M. Boscardin, F. Ficorella and G. Paternoster, "Development and First Parametric Characterization of High Fill-Factor Segmented LGADs with Trench Isolation.” 34th RD50 Workshop, 2019.

[22] G. Pellegrini, M. Baselga, M. Carulla, V. Fadeyev, P. Fernández-Martínez, M. Fernández et al., Recent technological developments on LGAD and iLGAD detectors for tracking and timing applications, Nuclear Instruments and Methods in Physics Research A 831 (2016) 24.

[23] M. Carulla, A. Doblas, D. Flores, Z. Galloway, S. Hidalgo, G. Kramberger et al., 50 um thin Low Gain Avalanche Detectors (LGAD) for timing applications, Nuclear Instruments and Methods in Physics Research A 924 (2019) 373.

[24] M. Mandurrino, R. Arcidiacono, M. Boscardin, N. Cartiglia, G. F. D. Betta, M. Ferrero et al., Demonstration of 200-, 100-, and 50-m Pitc hResistive AC-Coupled Silicon Detectors (RSD)With 100 \% Fill-Factor for 4D Particle Tracking, IEEE Electron Device Letters 40 (2019) 1780.

[25] G. Kramberger, M. Baselga, V. Cindro, P. Fernández-Martínez, D. Flores, Z. Galloway et al., Radiation effects in Low Gain Avalanche Detectors after hadron irradiations, Journal of Instrumentation 10 (2015) P07006.

[26] Z. Galloway, V. Fadeyev, P. Freeman, E. Gkougkousis, C. Gee, B. Gruey et al., Properties of HPK UFSD after neutron irradiation up to $6 e 15 \mathrm{n} / \mathrm{cm} 2$, Nuclear Instruments and Methods in Physics Research A 940 (2019) 19.

[27] M. Ferrero, R. Arcidiacono, M. Barozzi, M. Boscardin, N. Cartiglia, G. DallaBetta et al., Radiation resistant LGAD design, Nuclear Instruments and Methods in Physics Research A 919 (2019) 16.

[28] G. Kramberger, M. Carulla, E. Cavallaro, V. Cindro, D. Flores, Z. Galloway et al., Radiation hardness of gallium doped low gain avalanche detectors, Nuclear Instruments and Methods in Physics Research A 898 (2018) 53.

[29] E. Vilella, Recent depleted CMOS developments within the CERN-RD50 framework, Proceedings of Science (Vertex2019) (2020).

[30] M. Deveaux, Progress on the radiation tolerance of CMOS Monolithic Active Pixel Sensors, Journal of Instrumentation 14 (2019) R11001. 
[31] T. Wang, M. Barbero, I. Berdalović, C. Bespin, S. Bhat, P. Breugnon et al., Depleted fully monolithic CMOS pixel detectors using a column based readout architecture for the ATLAS Inner Tracker upgrade, Journal of Instrumentation 13 (2018) C03039.

[32] F. Palomo, M. Moll, J. Schwandt, E. Villani, Y. Gurimskaya and R. Millán, TCAD Device Simulations of Irradiated Silicon Detectors, Proceedings of Science (Vertex2019) (2020) .

[33] V. Eremin, N. Strokan, E. Verbitskaya and Z. Li, Development of transient current and charge techniques for the measurement of effective net concentration of ionized charges (Neff) in the space charge region of p-n junction detectors, Nuclear Instruments and Methods in Physics Research A 372 (1996) 388.

[34] G. Kramberger, V. Cindro, I. Mandić, M. Mikuž, M. Milovanović, M. Zavrtanik et al., Investigation of Irradiated Silicon Detectors by Edge-TCT, IEEE Transactions on Nuclear Science 57 (2010) 2294.

[35] P. Castro, M. Fernández, J. González, R. Jaramillo, M. Moll, R. Montero et al., “A novel Transient-Current-Technique based on the Two Photon Absorption process." 25th RD50 Workshop, 2014.

[36] M. Fernández, R. Jaramillo, M. Moll, R. Montero, R. Palomo, I. Vila et al., High resolution 3D characterization of silicon detectors using a Two Photon Absorption Transient Current Technique, Nuclear Instruments and Methods in Physics Research A, https://doi.org/10.1016/j.nima.2019.162865 (2019).

[37] M. Moll, Displacement Damage in Silicon Detectorsfor High Energy Physics, IEEE Transactions on Nuclear Science $\mathbf{6 5}$ (2018) 1561.

[38] F. Hartmann, Evolution of Silicon Sensor Technology in Particle Physics, 2nd edition, Springer International Publishing AG (2017).

[39] "Radiation effects at the LHC experiments and impact on operation and performance, 1. workshop." https://indico.cern.ch/event/695271/, 2018.

[40] "Radiation effects at the LHC experiments and impact on operation and performance, 2. workshop." https://indico.cern.ch/event/769192/, 2019.

[41] G. Kramberger, V. Cindro, I. Mandić, M. Mikuž and M. Zavrtanik, Charge collection studies on custom silicon detectors irradiated up to $1.6 e 17$ neq/cm2, Journal of Instrumentation 8 (2013) P08004.

[42] G. Kramberger, Reasons for high charge collection efficiency of silicon detectors at HL-LHC fluences, Nuclear Instruments and Methods in Physics Research A 924 (2019) 192.

[43] M. Huhtinen, Simulation of non-ionising energy loss and defect formation in silicon, Nuclear Instruments and Methods in Physics Research A 491 (2002) 194.

[44] M. Huhtinen and A. Manousos, "On the uncertainties of silicon hardness factors." Radiation effects at the LHC experiments and impact on operation and performance, 2019.

[45] Y. Gurimskaya, P. D. de Almeida, M. Fernández, I. Mateu, M. Moll, E. Fretwurst et al., Radiation damage in p-type EPI silicon pad diodes irradiated with protons and neutrons, Nuclear Instruments and Methods in Physics Research A, https://doi.org/10.1016/j.nima.2019.05.062 (2019) .

[46] M. Moll, Acceptor removal - Displacement damage effects involving the shallow acceptor doping of p-type silicon devices, Proceedings of Science (Vertex2019) (2020).

[47] P. D. de Almeida, Y. Gurimskaya, I. Mateu, M. Fernández and M. Moll, "Characterization of acceptor removal in silicon pad diodes irradiated by protons and neutrons.” 32nd RD50 Workshop, 2018. 
[48] A. Abada, FCC-hh: The Hadron Collider:Future Circular Collider Conceptual Design Report Volume 3, European Physical Journal Special Topics 228 (2019) 755-1107.

[49] V. Cindro, A. Gorišek, B. Hiti, G. Kramberger, I. Mandić, M. Mikuž et al., "First measurements with silicon detectors irradiated above 3e17 n/cm2." 32nd RD50 Workshop, 2018.

[50] F. Pitters, “The CLIC Detector Concept.” https://arxiv.org/pdf/1802.06008.pdf, 2018. 